\title{
Special issue on ultrafast spectroscopy: fundamentals
}

\author{
Alfred Leitenstorfer ${ }^{3}$, Keith A Nelson ${ }^{2}$ and Koichiro Tanaka ${ }^{1}$ \\ ${ }^{1}$ Department of Physics, Kyoto University, Oiwake, Kitashirakawa, Sakyo, Kyoto 606-8502, Japan \\ ${ }^{2}$ Department of Chemistry, Massachusetts Institute of Technology, Cambridge, MA, United States of \\ America \\ ${ }^{3}$ Department of Physics and Center for Applied Photonics, University of Konstanz, D-78457 Konstanz, \\ Germany \\ E-mail: kochan@scphys.kyoto-u.ac.jp and kanelson@mit.edu
}

\section{Introduction}

Over the last years, progress in the generation of electromagnetic waves ranging from the long-wave infrared (LWIR) to the Terahertz $(\mathrm{THz})$ frequency range has provided full control over the electric field, both in generation and detection, at extremely strong amplitudes ranging up to the multi-GV m${ }^{-1}$. This development has lead to the exploration of strong-field effects with long-wavelength pulses in physics, chemistry, biology and the engineering sciences. Since this region of the electromagnetic spectrum marks the boundary between the realms of electronics and optics, it is an especially rewarding subject for a broad range of studies. Thus the time was right to initiate a peer-reviewed collection of contributions concerning the state-of-the-art in: LWIR-THz generation using laser based and accelerator based approaches; the use of strong LWIR-THz fields to steer the motion of free electrons, drive rotations of molecules, vibrations of crystal lattices, the precession of spins and to explore transient states of matter.

The special issue 'ultrafast spectroscopy: fundamentals' provides an overview of some of the most important developments in this context, while at the same time indicating applications of these new developments. It is complemented by a special issue in the sister journal Journal of Physics D entitled 'ultrafast spectroscopy: applications'.

\section{Themes}

The contributions published in response to our broad call may be grouped under the following topics, at the same time highlighting the areas where high-field $\mathrm{THz}$ science currently develops the most efficient activities and impact:

\subsection{Generation of long-wave infrared and $\mathrm{THz}$ radiation}

Efficient sources for intense and high-quality radiation in the LWIR and $\mathrm{THz}$ frequency range still pose a considerable technological challenge. They are clearly representing the prerequisite for further scientific progress in this field. Several new concepts for proceeding in this direction have been published within this special issue, covering both broadband pulsed and narrowband multicycle emission. The underlying nonlinear media are either based on semiconducting materials $[1,2]$ or plasma generation [3].

\subsection{THz-based concepts for ultrashort electron bunches and particle acceleration}

Traditionally, electron and other particle beams have been controlled in free space by means of microwave electronics which is limited to the GHz frequency range. In order to go beyond the state-of-the art both in terms of temporal precision and peak energy, it is natural to proceed to higher frequencies in the adjacent $\mathrm{THz}$ region. This step, however, is a tedious one due to the technological challenges found when concepts from electronics can no longer be employed because of their limited bandwidth and on the other side, optical approaches also have to be strongly modified due to the long-wavelength and limited light-matter coupling at relatively low frequencies. Quite naturally, prospect for mastering these challenges is generating a wide range of new concepts and ideas in this context. In this special issue, design criteria for femtoand attosecond pulsed electron guns are worked out [4], aiming at applications both in real and reciprocal space. Subsequently, such electron bunches may then be accelerated into new parameter ranges by THz-enabled concepts [5-7], potentially providing a new dimension of performance and compactness for experiments in elementary particle physics, technology and applications. 


\subsection{Interaction of matter with strong and phase-controlled laser fields}

The advent of optical electromagnetic fields at extremely high amplitudes and in the few-cycle limit has enabled us to reach a completely new regime of light-matter interaction where no longer the average intensity but the precise temporal evolution of the wave form is relevant. Several prominent developments in this exciting context may be found in the special issue. First of all, the $\mathrm{THz}$ frequency range hosts the collective resonances of condensed matter and especially the complex excitations found in systems with strong electronic correlations. Here, this field allows the most direct access to control and manipulation of the fundamental degrees of freedom, adding important cornerstones to the many unanswered questions concerning these complex materials [7, 8]. In the case of bulk semiconductors and low-dimensional materials, electric field amplitudes may be applied by $\mathrm{THz}$ technology which are far beyond the ones sustained by the system under stationary biasing conditions, thus allowing to reach extreme regimes of hot carrier effects which may become relevant in future high-frequency and high-power devices $[9,10]$. In case of small molecules in the gas phase, it is the ultrafast tunneling of electrons and subsequent acceleration in the laser field which leads to emission of high optical harmonics and a new regime of quantum coherent electronic motion [11]. Finally, artificial metamaterials may be designed to harness also magnetic component of high-field $\mathrm{THz}$ radiation [12], thus providing a bridge towards precise control of spins and magnetic phenomena which are naturally linked to this frequency range.

\section{Conclusion}

In total, this special issue provides a fascinating insight into the many aspects that are currently being explored in connection with long-wavelength infrared and $\mathrm{THz}$ spectroscopy and technology at high-field amplitudes. There is a favorable balance between experimental and theoretical studies, indicating a continuous and healthy progress of the area towards scientific maturity. Still, the untouched possibilities out there seem to be almost unlimited and clearly, the papers in this collection are representing only a small fraction of what appears to be possible at this stage. Hopefully, this special issue will help to motivate a new generation of young and talented researchers to join this fascinating field with all its diversity, complexity, intellectual challenge and all the opportunities that are emerging from this situation.

\section{References}

[1] Nugraha P S et al 2018 Efficient semiconductor multicycle terahertz pulse source J. Phys. B: At. Mol. Opt. Phys. 51 094007

[2] Xu J et al 2018 Three-octave terahertz pulses from optical rectification of $20 \mathrm{fs}, 1 \mu \mathrm{m}, 78 \mathrm{MHz}$ pulses in GaP J. Phys. B: At. Mol. Opt. Phys. 51154002

[3] Bagley J D et al 2018 Laser-induced plasma generation of terahertz radiation using three incommensurate wavelengths J. Phys. B: At. Mol. Opt. Phys. 51144004

[4] Fallahi A and Kärtner F 2018 Design strategies for single-cycle ultrafast electron guns J. Phys. B: At. Mol. Opt. Phys. 51 144001

[5] Tibai Z et al 2018 Relativistic electron acceleration by focused THz pulses J. Phys. B: At. Mol. Opt. Phys. 51134004

[6] Sharma A et al 2018 Terahertz-driven wakefield electron acceleration J. Phys. B: At. Mol. Opt. Phys. 51204001

[7] Miyamoto T et al 2018 Control of electronic states by a nearly monocyclic terahertz electric-field pulse in organic correlated electron materials J. Phys. B: At. Mol. Opt. Phys. 51162001

[8] Kawakami Y et al 2018 Strong light-field effects driven by nearly single-cycle $7 \mathrm{fs}$ light-field in correlated organic conductors J. Phys. B: At. Mol. Opt. Phys. 51174005

[9] Shin H J et al 2018 Ultrafast nonlinear travel of hot carriers driven by high-field terahertz pulse J. Phys. B: At. Mol. Opt. Phys. 51144003

[10] Stein M et al 2018 Exciton ionization by $\mathrm{THz}$ pulses in germanium J. Phys. B: At. Mol. Opt. Phys. 51154001

[11] Iravani $\mathrm{H}$ et al 2018 Contribution of the pre-ionized $\mathrm{H}_{2}$ and the ionized $\mathrm{H}_{2}+$ subsystems to the HHG Spectra of $\mathrm{H}_{2}$ in intense laser fields J. Phys. B: At. Mol. Opt. Phys. 51074003

[12] Polley D, Hagström N Z, von Korff Schmising C, Eisebitt S and Bonetti S 2018 Terahertz magnetic field enhancement in an asymmetric spiral metamaterial $J$. Phys. B: At. Mol. Opt. Phys. 51224001 